\title{
Comparative Planetology of Kuiper Belt Dwarf Planets Enabled by the Near-Term Interstellar Probe
}

\author{
White paper to the 2023 Planetary Science Decadal Survey
}

Kirby D. Runyon ${ }^{1}$ (APL, 11100 Johns Hopkins Road, Laurel, MD, 240-228-5960, kirby.runyon@jhuapl.edu), Caitlin Ahrens ${ }^{2}$, Chloe B. Beddingfield ${ }^{3,4}$, Joshua T. S. Cahill ${ }^{1}$, Richard J. Cartwright ${ }^{3}$, Ian Cohen ${ }^{1}$, Bryan Holler ${ }^{5}$, Ted Stryk ${ }^{6}$, Peter Kollmann ${ }^{1}$, Edwin S. Kite $^{7}$, James T. Keane ${ }^{8}$, John F. Cooper", Chester "Sonny" Harman", Michele T. Bannister ${ }^{9}$, Kelsi N. Singer ${ }^{10}$, Alan Stern ${ }^{10}$, Abigail Rymer ${ }^{1}$, Noam Izenberg ${ }^{1}$, Andrew Annex ${ }^{11}$

1Johns Hopkins University Applied Physics Laboratory (APL), Laurel, MD; ${ }^{2}$ Arkansas Center for Space and Planetary Science; ${ }^{3}$ SETI Institute; ${ }^{4}$ NASA Ames Research Center; ${ }^{5}$ Space Telescope Science Institute ; ${ }^{6}$ Roane State Community College; ${ }^{7}$ University of Chicago; ${ }^{8}$ California Institute of Technology; ${ }^{8}$ University of Canterbury, New Zealand; ${ }^{9}$ NASA Goddard Space Flight Center; ${ }^{10}$ Southwest Research Institute; ${ }^{11}$ Johns Hopkins University

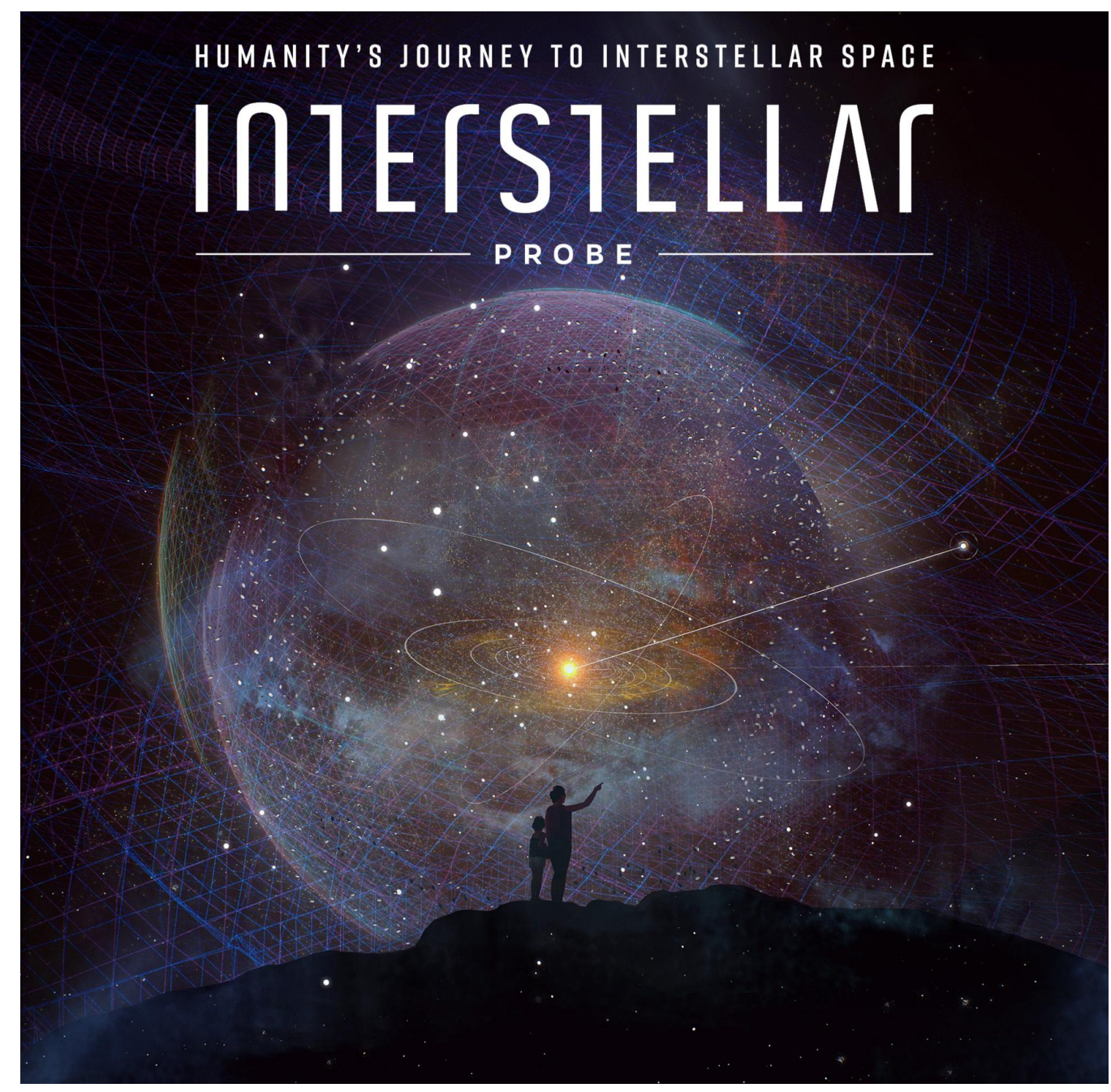


One-Sentence Summary. The near-term Interstellar Probe mission concept would enable flyby geoscience investigations of a Kuiper belt dwarf planet and its space environment, advancing comparative planetology beyond Neptune.

\section{Box 1. Comparative Planetology Questions for KBO Planets by Interstellar Probe}

- What fraction are geologically active? Is there ongoing plume, cryovolcanic, and/or tectonic activity? How are some planets able to remain active billions of years after their formation, as is the case for Pluto?

- What can the variability of landforms and compositions tell us about the various formation processes and evolutions of KBO planets?

- What fraction likely have or once had liquid water? If they had water, how long did it remain liquid before freezing? Was there a widespread process that extended the life of subsurface oceans, such as antifreeze? How is this related to the planet's window for habitability?

- How do their atmospheres interact with the solar wind/interstellar medium, and how does that interaction change based on the planet's changing solar distance?

- Can dwarf planets be used as analogs to infer properties of dwarf exoplanets?

\section{Introduction}

There are over 130 known Kuiper belt objects (KBOs) larger than $400 \mathrm{~km}$, and likely large enough to be dwarf planets (i.e., be round; Brown, 2020; IAU, 2006; Runyon et al., 2019a). In the coming decade, we will likely discover many more such planets with the Vera C. Rubin Observatory's Legacy Survey of Space and Time (formerly known as LSST) and the Nancy Grace Roman Space Telescope (formerly known as WFIRST). Dwarf planets in the Kuiper belt are the most common type of planet in the Solar System (and likely the most common type of exoplanet), far outnumbering terrestrial, giant, and satellite planets. Given their abundance, targeting one more of them for characterization offers a bonanza of new riches in comparative planetology.

KBO planets are diverse: Earth-based surveys show they have a broad range of bulk properties, including orbit, spin, composition, and shape. For instance, larger KBO planets host several volatile species (e.g., $\mathrm{N}_{2}, \mathrm{NH}_{3}, \mathrm{CH}_{4}, \mathrm{CO}$ ), which tend to be depleted on even smaller planets (e.g., Brown, 2012). Others, such as Haumea, are in hydrostatic equilibrium yet have elongated aspect ratios near 2:1 due to fast spins on the order of four hours. Even the "twin planets" of Pluto and Eris, which have similar sizes and masses, show very different albedos and compositions. Quaoar and Gonggong are examples of intermediate sizes and intermediate volatile abundance. Smaller planets, such as Orcus and Charon, reveal only water ice, which is likely mixed with ammoniahydrates (e.g., Brown, 2012).

While New Horizons and Voyager 2 provided the first reconnaissance of KBO planets (Pluto-Charon and Triton, respectively), the vast diversity of KBO planets has yet 
to be explored at close range. We argue that comparative planetology among KBO planets (1) should be a priority for future NASA missions and (2) may be partly addressed by a targeted KBO planet flyby via a probe leaving the Solar System.

Concordantly, NASA's Heliophysics Division is currently (as of mid-2020) investigating the near-term feasibility of a "pragmatic" (i.e., little-to-no technology development needed) interstellar probe to investigate the outer heliosphere and the local interstellar medium using current or near-term technologies with a launch-readiness date of January 1, 2030 (Brandt et al., 2020). This mission concept is termed Interstellar Probe (Brandt et al., 2020). Opportunistic reconnaissance of a KBO dwarf planet(s) by Interstellar Probe would enhance the science return from such a mission and would benefit from interdisciplinary, cross-divisional support within NASA's Science Mission Directorate (Zemcov et al., 2019; Mandt et al., 2020). For example, NASA's Planetary Science Division could treat a Heliophysics Division mission as a Mission of Opportunity (MoO) and provide funds and other support for instruments and operations that have planetary-specific applications.

The current concept for NASA's pragmatic Interstellar Probe would involve a 50year prime mission for a low-mass spacecraft launched on a powerful rocket (e.g., the Space Launch System), conducting a gravity assist by a giant planet, and leaving the Solar System significantly faster than Voyager 1's 3.6 AU/year on a primary mission to study the outer heliosphere's interaction with the local interstellar medium (McNutt et al., 2019; Brandt et al., 2020; Mandt et al., 2020). Compelling comparative planetology investigations await us among the KBO dwarf planets by Interstellar Probe as it exits the Solar System (Box 1).

To date, Triton, Pluto, and Charon are the only KBO planets visited by spacecraft. All three have transformed our view of planetary formation and evolution in different ways (e.g., Prockter et al., 2005; Nimmo \& Pappalardo, 2016; Beyer et al., 2019). Because of the significant challenges in reaching the Kuiper belt, Interstellar Probe could play a major role in advancing comparative planetology by reconnoitering one more KBO planet, such as Quaoar, Gonggong, or another of the $130+$ in the Kuiper belt.

A camera-instrumented Interstellar Probe could target high-resolution surface images, similar to New Horizons' flyby images of Pluto and Charon. Multispectral images and/or infrared spectrometers would reveal compositional variations, and magnetometers could reveal an intrinsic or remnant magnetic field, discussed below.

\section{Geomorphology}

Seeing a new planet "up close" for the first time is perhaps the most viscerally satisfying aspect of planetary exploration. Images that resolve landforms-typically at pixel scales better than a few kilometers or few $100 \mathrm{~m}$ per pixel—reveal geologic diversity and hint at the surficial and interior geophysical processes and timescales necessary to create those landforms. For instance, New Horizons' reconnaissance of Pluto and Charon revealed mountains, glaciers, craters, grabens, moats, frozen seas, possible 
cryovolcanoes, and even sand dunes (e.g., Stern et al., 2015; Moore et al., 2016; Telfer et al. 2018). The embayment relations between blocks and the surrounding smooth plains on Charon, together with the moon's hemispheric dichotomy, suggest past water and ice flows at the surface (Beyer et al., 2019). Drifted sand dunes on top of a convecting nitrogen ice glacier reveal sand-transporting winds on present-day Pluto (Telfer et al., 2018). Statistics of crater sizes on Pluto and Charon showed a paucity of craters smaller than $13 \mathrm{~km}$, revealing that impactors smaller than 1-2 km in diameter are scarce in the Kuiper belt (Singer et al., 2019). On Triton, recent and ongoing geyser-like eruptions deposit wind-blown dark material on the surface (Hansen et al., 1990) and Europa-like curvilinear ridges cross its surface (Prockter et al., 2005). Comparing all three instances of explored KBO planets shows surprising morphological and evolutionary diversity. Future surprises among other planets beckon further exploration in this region of the Solar System.

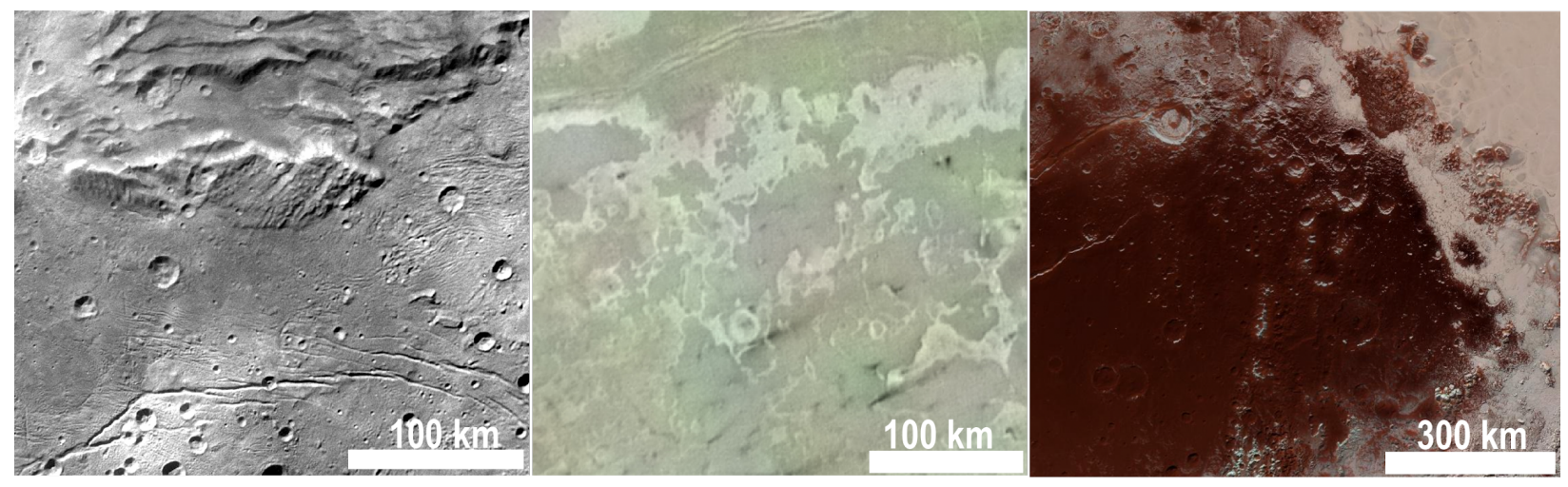

Figure 1. Charon (left), Triton (center), and Pluto (right) reveal the broad geophysical and evolutionary diversity across KBO planets. If Interstellar Probe is properly instrumented, flyby reconnaissance would reveal further diversity and advance comparative planetology. Pluto/Charon image credit: NASA/Southwest Research Institute/Johns Hopkins APL. Triton image credit: NASA/JPL.

\section{Geophysics}

The New Horizons mission demonstrated that even cold planets, far removed from the tidal heating of their icy moon cousins, can be geophysically active and possess subsurface oceans. If subsurface oceans past and present are common across the Kuiper belt, then the Kuiper belt may contain the legacy of a vast set of potentially habitable environments. Geophysical investigations are key to determining whether these planets are present-day ocean worlds and their history of habitability.

Even in a fast flyby, such as New Horizons or Interstellar Probe, there are a number of observations that can provide hints about the interior structure of a KBO planet. Cryovolcanic activity-such as active plumes on Triton (Hansen et al., 1990) or geologically recent volcanism on Pluto (Singer et al., 2019)—can expose subsurface materials and constrain the thermal evolution of the entire planet. Surface thermal 
anomalies, such as those observed at Enceladus (e.g., Spencer \& Nimmo, 2013), can also elucidate interior structure. Investigations of the distribution, orientation, and style of tectonic features can also reveal critical information on a world's dynamical history, as well as provide insight into the thermal and rheological properties of the interior. For example, the tectonics of Pluto implicate reorientation and the presence of a freezing subsurface ocean (Keane et al., 2016; Nimmo et al., 2017; Kamata et al., 2019). The phase space for the existence of subsurface oceans is currently so broad that even a fast flyby can strongly help in understanding the extent of habitability in this incredibly abundant population.

\section{Composition}

While images can reveal landforms and hint at geologic and geophysical processes, the surface composition can provide critical, complimentary insight on the planet's physical and chemical evolution. Visible and near-infrared imaging spectrometers provide data that can characterize connections between surface composition and geology. Understanding the composition of KBOs could provide key insights into how they formed and evolved. For example, compositional analysis of Charon's north polar region suggests that methane escaping from Pluto's atmosphere is being actively deposited on Charon and subsequently irradiated, forming the red refractory material observed by New Horizons (Grundy et al., 2016). On Pluto, hyperspectral data and compositional analysis have revealed putative cryovolcanic features that elucidate Pluto's thermal evolution (e.g., Dalle Ore et al., 2019; Cruikshank et al., 2019). Time-delay integration along narrow lines of clear and filtered co-added pixels is a proven method to acquire unsmeared multispectral images at high speed in the outer Solar System (e.g., Reuter et al., 2008; Runyon et al., 2019b). New Horizons' Ralph camera (Reuter et al., 2008) is an example of a high-technology-readiness-level (TRL) heritage instrument that could baseline the needed performance. Including spectrometers or multispectral cameras on Interstellar Probe would dramatically advance our knowledge of the connections between composition and geology on other planets.

\section{Atmosphere, Particles, and Fields}

All of the known KBO planets are currently embedded within the heliosphere, which determines the field, plasma, particle, dust, and ultraviolet environment. Knowledge of that environment is needed to understand the structure and composition of planetary atmospheres and surfaces (for example, tholin formation). KBOs at even larger distances from the Sun (e.g., Sedna and 2012 VP113) pass through the heliosheath boundary layer or very local interstellar environment, increasing their irradiance from galactic cosmic rays. Interstellar Probe instrumentation could systematically track the different effects that this has on surface and atmosphere composition. At times in the Sun's history, the heliosheath may be pushed inward. Thus, KBO planet depth profiles may not only reflect 
the history of solar activity but also provide insight into the Sun's journey through the galaxy.

The broad extent of Pluto's atmosphere hints at the possibility that other KBO planets could have atmospheres. A flyby of another KBO planet with Interstellar Probe, especially if carrying a magnetometer, would provide invaluable information on how geologic, atmospheric, interplanetary, and interstellar processes interact with small planets. Stern \& Trafton (2008) suggest that a number of processes driving atmospheric evolution for terrestrial planets, such as Jeans and hydrodynamic escape, operate on $\mathrm{KBO}$ planets. Constraining bulk composition and trace gas species in these atmospheres would provide a rich data set applicable to a broad swath of worlds and even exoplanets, and even without the detection of another atmosphere, characterizing a KBO planet's surface would put upper limits on the particles and radiation experienced by the planets, and by extension, the Solar System as a whole.

\section{Where to Go? Interstellar Probe's Trajectory Trade Space}

Interstellar Probe's direction of exit from our heliosphere into interstellar space would be dictated-to first order-by heliospheric constraints. Such constraints may include minimizing the distance to interstellar space and/or being able to characterize the heliosphere's shape externally, such as by flying near a "helioflank" away from the "nose" direction of interstellar gas inflow. With 130 known dwarf planets beyond Neptune, one of them will likely lie near a desired exit direction from our heliosphere, enabling flyby planetary exploration in a manner similar to New Horizons at Pluto-Charon (Bagenal et al., 2016). Two accessible and interesting currently known planets are Quaoar and Gonggong, both near the heliosphere's starboard helioflank $\left(\sim 300^{\circ}-330^{\circ}\right.$ ecliptic longitude) in the 2030s.

\section{Recommendations}

Interstellar Probe's high-speed, distantly-focused mission of exploration will captivate both professionals and the general public. We recommend that the pragmatic Interstellar Probe mission concept should be highly ranked by the Decadal Survey as a priority for 2023-2032. We recommend the Decadal Survey panel support KBO planet exploration via Interstellar Probe by acknowledging (1) Comparative planetology among the KBO planets can fundamentally inform Solar System formation, evolution, and windows of habitability; (2) the understanding of our own Solar System forms the basis for understanding exoplanetary systems, with implications for their habitability; (3) the Interstellar Probe mission concept would significantly advance comparative planetology on a uniquely bold and captivating mission; and (4) Interstellar Probe could create a new paradigm for interdisciplinary, cross-divisional support among the divisions in NASA's Science Mission Directorate (Mandt et al., 2020; Zemcov et al., 2019). 


\section{Bibliography}

Bagenal, F., et al. (2016). Pluto's interaction with its space environment: Solar wind, energetic particles, and dust. Science, 351, aad9045. DOI: 10.1126/science.aad9045.

Beyer, R. A., et al. (2019). The nature and origin of Charon's smooth plains. Icarus, 323, 16-32.

Brandt, P. C., et al. (2020). Interstellar Probe: Pushing the boundaries of space exploration, a white paper for the Solar \& Space Physics Decadal Survey 2023.

Brown, M. E. (2012). The compositions of Kuiper belt objects. Annual Review of Earth and Planetary Sciences, 40, 467-494.

Brown, M. E. (2020). How many dwarf planets are there in the outer solar system? http://web.gps.caltech.edu/ mbrown/dps.html. Accessed 29 May 2020.

Cheng, A. F., et al. (2009). Long-range reconnaissance imager on New Horizons. In New Horizons (pp. 189-215). Springer, New York, NY.

Cruikshank, D. P., et al. (2019). Recent cryovolcanism in Virgil Fossae on Pluto. Icarus, 330, 155-168.

Dalle Ore, C. M., et al. (2019). Detection of ammonia on Pluto's surface in a region of geologically recent tectonism. Science Advances, 5(5), eaav5731.

Grundy, W. M., et al. (2016). The formation of Charon's red poles from seasonally coldtrapped volatiles. Nature, 539(7627), 65-68.

Hansen, C. J., et al. (1990). Surface and airborne evidence for plumes and winds on Triton. Science, 250(4979), 421-424.

IAU (2006). Resolution 5B: Definition of a planet in the solar system. https://www.iau.org/static/resolutions/Resolution GA26-5-6.pdf. Accessed 29 May 2020.

Kamata, S., et al. (2019). Pluto's ocean is capped and insulated by gas hydrates. Nature Geoscience, 12(6), 407.

Keane, J. T., et al. (2016). Reorientation and faulting of Pluto due to volatile loading within Sputnik Planitia. Nature, 540(7631), 90.

Kivelson, M. G., et al. (2000). Galileo magnetometer measurements: A stronger case for a subsurface ocean at Europa. Science, 289(5483), 1340-1343.

Mandt, K. E., et al. (2020). Advancing space science requires NASA support for coordination between the science mission directorate communities. NAS white paper submitted to the Astrophysics, Planetary Science, and Heliophysics decadal survey committees.

McComas, D. J., et al. (2009). Global observations of the interstellar interaction from the Interstellar Boundary Explorer (IBEX). Science, 326(5955), 959-962.

McNutt, R. L. Jr., et al. (2019). Near-term interstellar probe: First step. Acta Astronautica, 162, 284-299. 
Moore, J. M., et al. (2016). The geology of Pluto and Charon through the eyes of New Horizons. Science, 351(6279), 1284-1293.

Nimmo, F., \& Pappalardo, R. T. (2016). Ocean worlds in the outer solar system. Journal of Geophysical Research: Planets, 121(8), 1378-1399.

Nimmo, F., et al. (2017). Mean radius and shape of Pluto and Charon from New Horizons images. Icarus, 287, 12-29.

Prockter, L. M., et al. (2005). A shear heating origin for ridges on Triton. Geophysical Research Letters, 32(14), L14202.

Reuter, D. C., et al. (2008). Ralph: A visible/infrared imager for the New Horizons Pluto/Kuiper belt mission. Space Science Reviews, 140(1-4), 129-154.

Runyon, K. D., et al. (2019a). Dwarf planets are planets, too: Planetary pedagogy after New Horizons. In Pluto System After New Horizons, abstract \#7016.

Runyon, K., et al. (2019b). Planetary imaging during fast flybys by Interstellar Probe. European Planetary Science Congress (EPSC) abstract, Vol. 13, EPSCDPS2019-716-1.

Ryan, K. J., et al. (2018, July). Design, fabrication, and test of a patterned optical filter array for the Europa Imaging System (EIS). In Advances in Optical and Mechanical Technologies for Telescopes and Instrumentation III (Vol. 10706, p. 1070655). International Society for Optics and Photonics.

Singer, K. N., et al. (2019). Impact craters on Pluto and Charon indicate a deficit of small Kuiper belt objects. Science, 363(6430), 955-959.

Spencer, J. R., \& Nimmo, F. (2013). Enceladus: An active ice world in the Saturn system. Annual Review of Earth and Planetary Sciences, 41, 693-717.

Stern, S. A., et al. (2015). The Pluto system: Initial results from its exploration by New Horizons. Science, 350(6258), aad1815.

Stern, S. A., \& Trafton, L. M. (2008). On the atmospheres of objects in the Kuiper belt (pp. 365-380). In The Solar System Beyond Neptune. University of Arizona Press, Tucson, AZ.

Telfer, M. W., et al. (2018). Dunes on Pluto. Science, 360(6392), 992-997.

Wei, H. Y., et al. (2010). Upper limits on Titan's magnetic moment and implications for its interior. Journal of Geophysical Research: Planets, 115(E10), E10007.

Zemcov, M., et al. (2019). Opportunities for astrophysical science from the inner and outer solar system. Astro2020: Decadal Survey on Astronomy and Astrophysics, science white papers, no. 60; Bulletin of the American Astronomical Society, Vol. 51, Issue 3, id. 60 . 